\title{
Determinación de la Longitud de Trabajo en Endodoncia. Implicancias Clínicas de la Anatomía Radicular y del Sistema de Canales Radiculares
}

\author{
Working Length Determination in Endodontics. Clinical Issues \\ of Dental Root and Root Canal Systems Morphology
}

\author{
Cynthia Rodríguez-Niklitschek ${ }^{*, * *}$ \& Gonzalo H. Oporto V.
}

RODRÍGUEZ-NIKLITSCHEK, C. \& OPORTO, V. G. H. Determinación de la longitud de trabajo en endodoncia. Implicancias clínicas de la anatomía radicular y del sistema de canales radiculares. Int. J. Odontostomat., 8(2):177-183, 2014.

RESUMEN: La endodoncia estudia la morfología y fisiología del sistema de canales de un diente. Su objetivo principal es prevenir lesiones pulpares y periodontales y tratar las ya instaladas. La determinación de la longitud de trabajo es una de las etapas más importantes del tratamiento de endodoncia. Imprecisiones en este proceso pueden favorecer la ocurrencia de accidentes y complicaciones postoperatorias. Esta revisión de la literatura describe la importancia de la anatomía dentaria aplicada a endodoncia y su estudio en cada población, además de presentar las principales técnicas para determinar la longitud de trabajo. La longitud de trabajo limita la preparación y obturación del canal radicular. Este límite debe ser la constricción apical. Diversos métodos de determinación han sido utilizados, donde el uso de localizadores apicales resulta ser el método más confiable. No obstante, es necesario conocer la morfología del sistema de canales en este proceso. Las longitudes radiculares referenciales a nivel mundial fueron publicadas por Ingle \& Bakland. No existen estudios que determinen la longitud de trabajo para cada diente en población sudamericana; tampoco los hay que determinen la longitud de trabajo en pacientes con localizadores apicales. Estudiar y conocer la anatomía radicular es un requisito fundamental para un tratamiento de endodoncia exitoso. Es esperable que existan diferencias entre las descripciones anatómicas dentarias clásicas y la anatomía dentaria de poblaciones específicas. Surge la necesidad de determinar parámetros de normalidad en cada población a nivel mundial.

PALABRAS CLAVE: endodoncia, raíz dentaria, tratamiento de canales, cavidad dentinaria pulpar, ápice dentario.

\section{INTRODUCCIÓN}

La Endodoncia es una especialidad de la odontología (reconocida como tal por la Asociación Dental Americana en 1963) que estudia la estructura, morfología y fisiología de las cavidades dentarias coronal y radicular que contienen a la pulpa dental y a su vez, trata la patología del complejo dentino-pulpar y de la región periapical (Fuentes et al., 2012). El objetivo de la endodoncia es prevenir lesiones pulpares y periodontales y tratar las ya instaladas, proporcionando el sustrato dentario para devolver forma y función perdidas, a través de la rehabilitación oral (Fuen- tes et al.). Para esto, es necesario realizar una minuciosa limpieza mecánica y química de la cámara pulpar y del sistema completo de canales radiculares (Al-Qudah \& Awawdeh, 2009; Chandra et al., 2011; De Moor et al., 2004; Huang et al., 2010a; Jarad et al., 2011; McDonald \& Hovland, 1990) los cuales, ya preparados y desinfectados, deben obturarse completamente con un material de relleno inerte y biocompatible. Luego, el diente debe ser restaurado adecuadamente, para asegurar su sellado coronal e impedir la filtración bacteriana (De Moor et al.).

\footnotetext{
Programa de Magíster en Odontología, Facultad de Odontología, Universidad de La Frontera, Temuco, Chile

* Escuela de Odontología, Cátedra de Endodoncia, Universidad Mayor sede Temuco, Temuco, Chile.

*** Departamento de Odontología Integral Adultos, Facultad de Odontología, Universidad de La Frontera, Temuco, Chile.

*** Centro de Biología Molecular y Farmacogenética, Departamento de Ciencias Básicas, Facultad de Medicina, Universidad de La Frontera, Temuco, Chile.
} 
La terapia endodóntica propiamente tal consiste en la extirpación de la pulpa presente en la cavidad dentaria cameral y los canales radiculares, desinfectar y conformar dichos canales y luego rellenarlos con un material biocompatible, con el fin de mantener el diente en la cavidad oral (Fuentes et al.). El procedimiento endodóntico incluye varias etapas: diagnóstico, trepanación y acceso a las cavidades dentarias pulpares de la corona y raíces, determinación de la longitud de trabajo de los canales radiculares (conductometría), instrumentación biomecánica o quimiomecánica (IBM ○ IQM), conometría y obturación radicular. La determinación de la longitud de trabajo es una de las etapas más importantes del tratamiento de endodoncia y es también uno de los pasos preponderantes en el éxito de la terapia.

Estudiar y conocer la anatomía radicular a cabalidad es un requisito fundamental para conseguir un tratamiento de endodoncia exitoso (Abella et al., 2012). Varios son los autores que han estudiado la longitud radicular de los diferentes dientes (Ingle \& Bakland, 2004; Martínez, 1998). A partir de estas mediciones se definió que el límite ideal de la obturación de los canales radiculares debe estar a 1 $\mathrm{mm}$ del ápice radicular (desde coronal a apical) (Fuentes et al.). Las mediciones obtenidas a partir de las investigaciones señaladas son utilizadas actualmente como medidas de referencia para la realización de tratamientos de endodoncia en muchos países del mundo; la mayoría de estos estudios fueron desarrollados en dientes extraídos y ninguno de ellos en población sudamericana. Por otra parte, no existen investigaciones que determinen la longitud de trabajo para los canales radiculares de los diferentes dientes, sino que sólo establecen una medida anatómica de la longitud radicular y la longitud total de cada diente.

En base a lo señalado, surge la importancia de conocer las implicancias clínicas que puede tener la morfología radicular y del sistema de canales en la práctica clinica de la endodoncia, especialmente en la determinación de la longitud de trabajo y del límite de obturación.

Esta investigación expone una revisión de la literatura en relación a la implicancia clínica de la anatomía dentaria y del sistema de canales radiculares en el éxito de los tratamientos de endodoncia.
Determinación de la longitud de trabajo. Una de las etapas más importantes y críticas de la terapia endodóntica es la conductometría. Este procedimiento tiene por objetivo obtener una medida de longitud, que corresponde a "la distancia desde un punto de referencia coronal hasta el punto donde termina la preparación y obturación del canal radicular" (Martínez). La determinación de una longitud de trabajo precisa es uno de los procedimientos más importantes de la terapia endodóntica, y es un factor clave para el éxito del tratamiento (Özsezer et al., 2007). La limpieza, conformación y obturación del sistema de canales radiculares no pueden lograrse con precisión a menos que se determine con exactitud esta medida. Establecer una longitud de trabajo adecuada es imprescindible para poder llevar a cabo un tratamiento exitoso; de no obtenerse inicialmente de manera correcta, todo el esfuerzo posterior en la consecución del éxito terapéutico puede ser infructuoso (Martínez). La determinación errada de la longitud de trabajo podría dar lugar a una medición demasiado larga y conducir la preparación más allá de la constricción apical, provocando sobre instrumentación y sobre obturación. A su vez, también podría llevar a la conformación hasta un punto anterior a la constricción apical, resultando así una limpieza y un relleno insuficiente del canal radicular (Özsezer et al.). Por su forma estrecha, la constricción apical provee una resistencia natural al empacamiento del material obturador y debe respetarse (McDonald \& Hovland).

La conductometría establece la extensión apical de la instrumentación y el último nivel apical de la obturación del canal radicular. La preparación y obturación del canal radicular debe finalizar a nivel de la unión cemento-dentinaria, confinada al interior del sistema de canales radiculares (Jarad et al.; McDonald \& Hovland). La unión cemento-dentinaria se define como "el punto más apical de la pulpa dental" (Martínez), y corresponde al punto donde deja de haber dentina y el canal se continúa con paredes de cemento. El objetivo de determinar una longitud de trabajo es lograr preparar y obturar los canales radiculares lo más cerca posible de este punto, idealmente a nivel de la constricción apical, área del canal radicular que presenta el menor diámetro y es el punto de unión entre el tejido pulpar y el tejido periodontal (Jarad et al.). La unión cementodentinaria puede o no coincidir con la constricción apical; esta última debe ser el límite de la preparación y de la obturación radicular. La ubicación de la constricción apical varía considerablemente y su re- 
lación con la unión cemento-dentinaria también es variable y puede ser de hasta $3 \mathrm{~mm}$ más alta en un lado del canal, en comparación con el otro. Por otra parte, la unión cemento-dentinaria no puede ser identificada clínicamente (Somma et al., 2012). La constricción apical se ubica generalmente entre 0,5 a 2 $\mathrm{mm}$ del ápice dentario radiográfico (Jarad et al.), y hay estudios que relacionan la posición de la unión cemento-dentinaria y la constricción apical, mostrando esta última siempre más coronal a la primera (Somma et al.).

Consecuencia de una determinación de longitud de trabajo errónea. La determinación imprecisa de la longitud de trabajo puede favorecer la ocurrencia de accidentes endodónticos, como perforación apical y sobre obturación, las cuales son generalmente acompañadas de dolor postoperatorio. Por otra parte, el inicio de la reparación periapical puede prolongarse en el tiempo, aumentando así el número de fracasos por regeneración incompleta de los tejidos periapicales. Otro riesgo presente frente a una longitud de trabajo incorrecta es la instrumentación incompleta y la obturación deficiente del canal radicular, con todos los problemas que ello trae (reagudización de la infección y de los síntomas, reinfección del canal radicular, aparición de lesiones apicales, dolor persistente debido a la inflamación de tejido pulpar no eliminado). Además, puede formarse un escalón antes del ápice, lo que podría imposibilitar un retratamiento exitoso, de ser necesario en el futuro. El hueso, cemento y ligamento periodontal pueden transformarse en elementos activos con gran capacidad de recambio, lo que los hace particularmente aptos para el restablecimiento de las condiciones anatomo-fisiológicas normales a nivel del periápice (Martínez). Esta es una de las razones por las cuales no es conveniente invadir más allá de la constricción apical al instrumentar o al obturar los canales radiculares, a fin de mantener esta zona intacta con todas sus potencialidades de reparación. Una obturación radicular finalizada en la constricción apical proporciona las condiciones óptimas para la reparación, con un contacto mínimo entre el material de relleno y el tejido apical, reduciendo de este modo la destrucción de tejidos, evitando la persistencia de respuestas inflamatorias y reacciones a cuerpo extraño (Somma et al.).

\footnotetext{
Métodos para la determinación de la longitud de trabajo. A lo largo de los años, las técnicas más habituales para determinar la longitud de trabajo han sido la sensación táctil y la técnica radiográfica
}

(McDonald \& Hovland; Shanmugaraj et al., 2007), sin embargo, ninguna de las dos técnicas puede determinar la ubicación de la constricción apical (Özsezer et al.; Shanmugaraj et al.). La sensación táctil es altamente inespecífica, más aún en canales de raíces con ápice inmaduro, con curvatura excesiva o que se van estrechando a lo largo de todo su recorrido (Shanmugaraj et al.). Por otra parte, la única información segura que nos ofrece la radiografía es la localización del ápice radiográfico, definido como la porción más apical del diente en una radiografía (Jarad et al.), lo cual está determinado también por la interpretación que le da el clínico (McDonald \& Hovland; Shanmugaraj et al.). El ápice anatómico puede coincidir o no con el foramen apical, que es la zona en donde el canal se abre a la superficie radicular contactando con el ligamento periodontal. Dado que frecuentemente el foramen apical no se localiza en el ápice radiográfico, sino lateralmente a este, la ubicación de la lima a esta longitud produciría muchas veces sobre instrumentación y sobre obturación.

Con frecuencia se puede observar en la conductometría radiográfica el lugar por donde la lima sale de la raíz cuando el foramen apical está ladeado hacia mesial o distal, o bien cuando coincide con el ápice anatómico. Sin embargo, cuando el foramen se encuentra en dirección vestibular o lingual, ocurre una superposición de su imagen con el ápice, lo cual puede ocasionar un error de interpretación radiográfica (Martínez). Un estudio con dientes extraídos determinó que, al medir los canales radiculares con limas colocadas a la longitud dada por la ubicación del ápice radiográfico, sólo un $82 \%$ coincidió con el foramen apical real (Olson et al., 1991).

En la actualidad, existen herramientas que permiten obtener una medición de la longitud de trabajo más confiable; una de ellas es el localizador apical. El concepto de la medición de la longitud de trabajo de los canales radiculares utilizando un dispositivo de localización apical surgió en 1942, y fue descrito por Suzuki (Kqiku et al., 2011), mientras que la resistencia eléctrica entre el ligamento periodontal y la mucosa oral fue descrita por Sunada en 1962 (Jarad et al.; Kqiku et al.).

El concepto básico para todos los dispositivos de medida de longitud electrónicos se basa en que los tejidos humanos tienen ciertas características que pueden ser modeladas por una combinación de com- 
ponentes eléctricos. Por lo tanto, mediante la medición de las propiedades eléctricas, tales como la resistencia y la impedancia, puede ser posible detectar el extremo del canal radicular (Nekoofar et al., 2006). El sistema de canales radiculares está rodeado por la dentina y el cemento, que son aislantes de la corriente eléctrica. En el foramen apical menor (en donde comienza la constricción apical), las sustancias conductoras que están dentro de la cavidad del canal radicular (tejido, fluido) se conectan eléctricamente al ligamento periodontal que es en sí mismo un conductor de la corriente eléctrica. Por lo tanto, la dentina, junto con el tejido y el fluido dentro del canal, forma una resistencia, cuyo valor depende de sus dimensiones y de su resistencia inherente.

Cuando una lima de endodoncia penetra en el interior del canal y se acerca al foramen apical menor, la resistencia entre la lima de endodoncia y el foramen disminuye, porque la longitud efectiva del material resistivo (dentina, tejido, fluido) disminuye (Nekoofar et al.). Así, se han desarrollado varios medios electrónicos que utilizan una variedad de otros principios para detectar el extremo del canal radicular. Sunada determinó que la resistencia eléctrica entre un instrumento que tocaba la membrana periodontal (por una parte) y la mucosa oral (por otra) era un valor constante, con independencia de la edad del paciente, del diámetro del canal o de la forma de los dientes (McDonald \& Hovland). Desde este descubrimiento, varios localizadores apicales electrónicos han sido introducidos en el mercado. Mientras que el dispositivo más simple medía la resistencia, otros dispositivos pueden medir la impedancia usando alta frecuencia, dos frecuencias, o varias frecuencias. Además, algunos sistemas utilizan baja frecuencia de oscilación o una tensión (método del gradiente) para detectar el extremo del canal. Los primeros localizadores electrónicos en aparecer determinaban la posición del ápice a través de la medición del valor de resistencia descrito por Sunada (Jarad et al; Kqiku et al.). Muchos de estos localizadores demostraron ser extremadamente precisos en canales secos, sin embargo, su uso fue limitado debido a su falta de precisión en canales húmedos o con presencia de tejido pulpar, sangre o restos de irrigantes (Özsezer et al.). En estas situaciones, dichos localizadores registraban que el ápice había sido alcanzado, cuando en realidad la punta de la lima tocaba la solución que completaba el circuito eléctrico (McDonald \& Hovland). Los localizadores apicales antiguos emitían una leve corriente eléctrica continua y medían la resistencia de los tejidos al paso de ella. Los actuales emiten también una corriente leve, pero de tipo alterno y miden la impedancia entre el tejido apical y la mucosa bucal. La resistencia, así como la impedancia miden la dificultad que tiene la electricidad para pasar a través de algún material. En general, cuando la corriente eléctrica es continua, se habla de resistencia; si la corriente es alterna, se habla de impedancia. Los tejidos blandos conducen la electricidad con relativa facilidad, en cambio los tejidos duros tienden a oponer resistencia al paso de la corriente eléctrica actuando como aislante. De esta manera, si por un lado tenemos un electrodo colocado en el labio, es decir, mucosa bucal, y por otro lado un electrodo en el diente a examinar, al activar el sistema del localizador, tendremos el paso de una corriente alterna muy pequeña entre los dos electrodos. Al comenzar, si se toca con una lima el esmalte y posteriormente la dentina, los valores de impedancia serán muy altos, pero al ingresar al interior del canal radicular y recorrerlo (tercio cervical, tercio medio y tercio apical), los valores de impedancia irán disminuyendo progresivamente. Finalmente, cuando la lima toque a través del canal el tejido periodontal, los valores caerán bruscamente ya que se habrá cerrado el circuito entre la mucosa oral y el periodonto. Así, a través del uso de la corriente alterna y la determinación de la impedancia, se puede determinar el área donde termina el canal radicular y comienzan los tejidos periapicales.

El uso del localizador apical se ha masificado durante los últimos años, especialmente con la introducción de los localizadores de tercera y cuarta generación. Los localizadores apicales de tercera generación tienen microprocesadores más potentes y son capaces de procesar cuocientes matemáticos y calcular algoritmos, necesarios para dar lecturas precisas. Los de cuarta generación no procesan la información de impedancia como un algoritmo matemático, sino que toman la resistencia y capacitancia por separado, y los comparan con una base de datos para determinar la distancia al ápice radicular (Özsezer et al.).

Los localizadores apicales basados en múltiples frecuencias se han desarrollado para aumentar aún más la precisión. Se basan en el concepto de medir las características de impedancia con más de dos frecuencias. Los localizadores modernos funcionan mediante el uso de diferentes frecuencias, determinando la relación entre los diferentes potenciales eléctricos proporcionales a cada impedancia (Özsezer et al.). Además, tienen la ventaja de no 
necesitar secar el canal radicular antes de usarlo, porque trabajan en la presencia de electrolitos. Un ejemplo de esta generación de localizadores apicales es ProPex® (Dentsply, Maillefer, Ballaigues, Suiza). Este aparato permite la medición del canal radicular en cualquier condición (presencia de hipoclorito de sodio en el canal, canal seco, canal húmedo, etc.), como resultado de su tecnología de multifrecuencia.

El funcionamiento de ProPex® se basa en el mismo principio de los otros dispositivos modernos que utilizan múltiples frecuencias para determinar la longitud del canal radicular; una característica importante de ProPex® es que el cálculo se basa en la energía de la señal, mientras que los otros localizadores de ápice utilizan la amplitud de la señal (Özsezer et al.).

El localizador apical electrónico elimina muchos de los problemas asociados con los métodos radiográficos (Shanmugaraj et al.). Su principal ventaja es que puede medir la longitud del canal radicular hasta el final de la unión cemento-dentinaria. El desarrollo de dispositivos electrónicos de medición de longitud del canal radicular ha ayudado a hacer la evaluación de la longitud de trabajo más precisa y predecible (Somma et al.). Se considera que es un método fácil y rápido, sin requisitos de exposición de rayos $x$ (excepto cuando la radiografía se utiliza como complemento, para confirmar la medida de longitud indicada por el localizador apical). Sin embargo, no se debe olvidar que su precisión está influenciada por condiciones eléctricas del canal y es inexacta en dientes con ápice muy abierto y canales calcificados (Shanmugaraj et al.). Por otra parte, los localizadores apicales no localizan la constricción apical precisamente, sino más bien, detectan un área entre el foramen apical menor (constricción apical) y el foramen apical mayor (foramen apical) en condiciones clínicas (Somma et al.).

Anatomía Radicular. Frente a todos los riesgos que implica instrumentar un canal radicular sin una medición precisa de la longitud de trabajo de este, resulta importante que el odontólogo conozca a cabalidad la anatomía del diente a tratar y sus posibles variaciones anatómicas (Al-Qudah \& Awawdeh; Huang et al., 2010b; Martínez).

Así, la comprensión de la anatomía radicular contribuirá a mejorar los resultados de los tratamientos y es fundamental para lograr un tratamiento de endodoncia exitoso (Abella et al.). Al respecto, son varios los autores que han estudiado la longitud de los diferentes dientes; Weine en 1991, realizó un estudio para determinar la longitud de los dientes en nueve mil casos. La longitud media se determinó desde la punta de la cúspide hasta el ápice radicular. En 1987, Ingle y Taintor realizaron un estudio para determinar las longitudes media, máxima y mínima de todos los grupos de dientes (Martínez). Posteriormente Ingle, publicó las longitudes radiculares de todos los dientes humanos, basándose en los resultados de estudios de diversos autores (Ingle \& Bakland).

Actualmente, la longitud radicular promedio utilizada como referencia para tratamientos de endodoncia en Chile y en otros países del mundo, está determinada por los resultados obtenidos a partir de los estudios mencionados, la mayoría desarrollados en dientes extraídos y ninguno de ellos realizado en población sudamericana. A su vez, no existen estudios que determinen la longitud de trabajo para cada canal radicular, sino que los estudios existentes definen la longitud de cada raíz, en cada uno de los dientes, desde un límite coronal hasta el ápice radicular. En base a estas mediciones disponibles, se estableció como consenso que la longitud de trabajo para cada canal radicular y la obturación radicular deben limitarse a un punto distante de 1 $\mathrm{mm}$ al ápice radicular (desde coronal a apical) (Fuentes et al.). Parece relevante analizar este antecedente, especialmente al considear que en la actualidad, cada vez más, la determinación de la longitud de trabajo se realiza utilizando localizadores apicales; resulta paradojal que se mantenga este consenso como referencia aceptada para un procedimiento clínico y no se reformulen estas referencias en base a estudios de mediciones realizadas en dientes que requieren tratamiento de endodoncia, donde la longitud de trabajo se obtiene con localizadores apicales.

Proyecciones. La determinación adecuada de la longitud de trabajo es un procedimiento fundamental en la obtención de un pronóstico favorable de la terapia endodóntica. Para lograr éxito en este procedimiento, es necesario poseer profundos conocimientos de la morfología radicular y del sistema de canales en cada diente, además de conocer los métodos de obtención de esta medida y manejar antecedentes en cuanto a qué método alcanza una longitud de trabajo que se relaciona con un tratamiento clínicamente exitoso.

Es sabido que la anatomía dentaria, y 

canales radiculares. Int. J. Odontostomat., 8(2):177-183, 2014.

específicamente la anatomía radicular, puede presentar variaciones de acuerdo a la población analizada. Hasta la fecha, un número importante de investigaciones han informado que los sistemas radiculares varían según el origen étnico del individuo, y estas variaciones podrían estar determinadas genéticamente (Al-Qudah et al.; Zhang et al., 2011). Luego, es esperable que existan diferencias entre las descripciones anatómicas dentarias clásicas y la anatomía dentaria de poblaciones específicas alrededor del mundo. Por lo anterior, surge la necesidad de determinar parámetros de normalidad atingentes a cada población de modo de establecer rangos de normalidad en ellos y no presumirlos a partir de estudios rea- lizados en grupos específicos de personas (Ingle \& Bakland), que no necesariamente pueden ser extrapolados a todos los individuos a nivel mundial.

En base a lo enunciado, y a las eventuales diferencias en la anatomía dentaria y en el sistema de canales que existiría en los diversos grupos de personas alrededor del mundo, parece necesario abrir el campo de la investigación de la morfología dentaria aplicada a la práctica clínica de la endodoncia en población americana, de manera de aportar nuevos datos e incrementar las tasas de éxito de estos procedimientos odontológicos, además de establecer las bases para futuras investigaciones

RODRÍGUEZ-NIKLITSCHEK, C. \& OPORTO, V. G. H. Working length determination in endodontics. Clinical issues of dental root and root canal systems morphology. Int. J. Odontostomat., 8(2):177-183, 2014.

ABSTRACT: Endodontics is a dentistry specialty concerned with the study of root canal morphology and physiology, besides treating pathologies affecting root canal system and periapical region. Determining working length is one of the most important stages of endodontic therapy. Inaccuracies in this process may contribute to occurrence of accidents and postoperative pain. This literature review develops the importance of studying dental anatomy applied to endodontics, as well as the importance of performing research in this field in diverse populations worldwide, and discuss the main techniques to determine working length. Limits of root canal preparation and obturation are given by working length. This limit is the apical constriction. Several methods have been used to determine this, where apex locators are the most reliable tool, it is however, necessary to know root canal system morphology in this process. Referential teeth root lengths used nowadays in endodontics were published by Ingle \& Bakland. There are no studies determining if teeth length in South American population, are in agreement with Ingle's data, nor is there any research determining working length with apex locators. In order to achieve a successful endodontic therapy it is necessary to know teeth root morphology as well as root canals systems anatomy. It is expected to discover differences between classical dental anatomical descriptions and dental morphology of specific populations. There is a need to determine root canal system parameters of normality in populations worldwide.

KEY WORDS: endodontics, root canal therapy, dental pulp cavity, tooth root, tooth apex.

en esta área en diversas latitudes.

\section{REFERENCIAS BIBLIOGRÁFICAS}

Abella, F.; Patel, S.; Durán-Sindreu, F.; Mercadé, M. \& Roig, M. Mandibular first molars with disto-lingual roots: review and clinical management. Int. Endod. J., 45(11):963-78, 2012.

Al-Qudah, A. A. \& Awawdeh, L. A. Root and canal morphology of mandibular first and second molar teeth in a Jordanian population. Int. Endod. J., 42(9):77584, 2009.

Chandra, S. S.; Chandra, S.; Shankar, P. \& Indira, R. Prevalence of radix entomolaris in mandibular permanent first molars: a study in a South Indian population. Oral Surg. Oral Med. Oral Pathol. Oral Radiol. Endod., 112(3):e77-82, 2011.

De Moor, R. J.; Deroose, C. A. \& Calberson, F. L. The radix entomolaris in mandibular first molars: an endodontic challenge. Int. Endod. J., 37(11):789-99, 2004.

Fuentes, R.; Rodríguez, C. \& Pérez, L. Endodoncia: Conceptos Básicos. Texto guía para el aprendizaje de endodoncia de pregrado y postgrado. Temuco, Universidad Mayor, 2012.

Huang, C. C.; Chang, Y. C.; Chuang, M. C.; Lai, T. M.; Lai, J. Y.; Lee, B. S. \& Lin, C. P. Evaluation of root and canal systems of mandibular first molars in Taiwanese individuals using cone-beam computed tomography. J. Formos. Med. Assoc., 109(4):303-8, 2010 a.

Huang, R. Y.; Cheng, W. C.; Chen, C. J.; Lin, C. D.; Lai, T. M.; Shen, E. C.; Chiang, C. Y.; Chiu, H. C. \& Fu, E. Three-dimensional analysis of the root morphology of mandibular first molars with distolingual roots. Int. Endod. J., 43(6):478-84, 2010 b. 
RODRÍGUEZ-NIKLITSCHEK, C. \& OPORTO, V. G. H. Determinación de la longitud de trabajo en endodoncia. Implicancias clínicas de la anatomía radicular y del sistema de canales radiculares. Int. J. Odontostomat., 8(2):177-183, 2014.

Ingle, J. I. \& Bakland, L. K. Endodoncia. 5a ed. México, D.F., McGraw-Hill, 2004. pp.427-75.

Jarad, F. D.; Albadri, S.; Gamble, C.; Burnside, G.; Fox, K.; Ashley, J. R.; Peers, G. \& Preston, A. J. Working length determination in general dental practice: a randomised controlled trial. Br. Dent. J., 211(12):595-8, 2011.

Kqiku, L. \& Städtler, P. Radiographic versus electronic root canal working length determination. Indian J. Dent. Res., 22(6):777-80, 2011.

Martínez, M. Aportación metodológica a la determinación de la longitud de trabajo en endodoncia. Tesis de Doctor en Odontología. Valencia, Universidad de Valencia, 1998. pp.10,11,13-15,36.

McDonald, N. J. \& Hovland, E. J. An evaluation of the Apex Locator Endocater. J. Endod., 16(1):5-8, 1990.

Nekoofar, M. H.; Ghandi, M. M.; Hayes, S. J. \& Dummer, P. $M$. The fundamental operating principles of electronic root canal length measurement devices. Int. Endod. J., 39(8):595-609, 2006.

Olson, A. K.; Goerig, A. C.; Cavataio, R. E. \& Luciano, J. The ability of the radiograph to determine the location of the apical foramen. Int. Endod. J., 24(1):28-35, 1991.

Özsezer, E.; Inan, U. \& Aydin, U. In vivo evaluation of ProPex electronic apex locator. J. Endod., 33(8):9747, 2007.

Shanmugaraj, M.; Nivedha, R.; Mathan, R. \& Balagopal, S. Evaluation of working length determination methods: an in vivo / ex vivo study. Indian J. Dent. Res., 18(2):602, 2007

Somma, F.; Castagnola, R.; Lajolo, C.; Paternò Holtzman, L. \& Marigo, L. In vivo accuracy of three electronic root canal length measurement devices: Dentaport ZX, Raypex 5 and ProPex II. Int. Endod. J., 45(6):552-6, 2012.

Zhang, R.; Wang, H.; Tian, Y. Y.; Yu, X.; Hu, T. \& Dummer, P. M. Use of cone-beam computed tomography to evaluate root canal and canal morphology of mandibular molars in Chinese individuals. Int. Endod. J.,
44(11):990-9, 2011.

Dirección de correspondencia:

Cynthia Rodríguez Niklitschek

Cirujano Dentista, Especialista en Endodoncia

Docente Cátedra de Endodoncia Universidad Mayor

Sede Temuco.

Avenida Alemania 0281, Temuco

CHILE

Email: cynthia.rodriguez@mayor.cl

Recibido : 30-06-2013

Aceptado: 19-05-2014 